\title{
Continuous - Time Active Filter Design Using Signal - Flow Graphs
}

\author{
Martin Minarcik, Kamil Vrba \\ Department of Telecommunications, Faculty of Electrical Engineering and Communication, \\ Brno University of Technology, \\ Purkynova 118, 61200 Brno, Czech Republic \\ xminar08@stud.feec.vutbr.cz, vrbak@feec.vutbr.cz
}

\begin{abstract}
This article deals with active frequency filter design using signalflow graphs. The procedure of multifunctional circuit design that can realize more types of frequency filters is shown. To design a new circuit the Mason Coates graphs with undirected self-loops have been used. The voltage conveyors whose properties are dual to the properties of the well-known current conveyors have been used as the active element.
\end{abstract}

Keywords: frequency filter design, multifunctional frequency filters, signalflow graps.

\section{Introduction}

The voltage feedback amplifier is the most often used part in the area of signal processing. Nowadays, new active elements such as transconductance amplifiers, transadmittance amplifiers, voltage and current conveyors come to be used. The utilization of circuits with the new active elements offers many advantages compared to structures with the voltage feedback amplifiers. The internal structures of moderm active elements are realized with the help of simple building blocks and that is why the signal with a wide frequency range can processed. A big group of new active elements is formed by current and voltage conveyors. At our department the signal flow graph method has been used to design new circuits with voltage conveyors.

Signal - flow graphs have been used in many areas of engineering. In the area of signal processing the signal flow - graphs were used especially as an effective instrument for a quick analysis of linear circuits. Nowadays this problem is solved mainly by computer technology and suitable computer programmes so that signal flow graphs are rarely used in signal processing today [1], [2]. However they are a suitable aid in the design of new circuits using nontraditional parts. 


\section{Signal - flow graph of voltage conveyor}

In 1999 one type of voltage conveyor was presented [3]. The other types or generations of voltage conveyors have been developed in our department [4]. The voltage conveyor properties were derived from the current conveyor properties by interchanging the currents and the voltages in the describing equations. The basic types of voltage conveyors are three - port active elements with one high impedance input $\mathbf{x}$, low - impedance input $\mathbf{y}$, and voltage output $\mathbf{z}$. Twelve types of voltage conveyors can be defined according to the way voltages and currents are transferred between separate ports of the voltage conveyor. For their common definition the generalized voltage conveyor - GVC (Fig.1.) can be used.

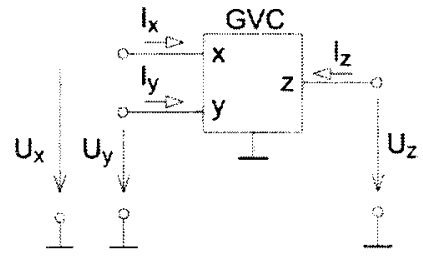

Fig. 1. Schematic representation of the GVC

The currents and voltages relations between GVC ports are described by the following matrix equation

$$
\left(\begin{array}{c}
I_{\mathrm{x}} \\
U_{\mathrm{y}} \\
U_{\mathrm{z}}
\end{array}\right)=\left(\begin{array}{lll}
0 & \alpha & 0 \\
\beta & 0 & 0 \\
\gamma & 0 & 0
\end{array}\right) \cdot\left(\begin{array}{c}
U_{\mathrm{x}} \\
I_{\mathrm{y}} \\
I_{\mathrm{z}}
\end{array}\right),
$$

where $\alpha, \beta, \gamma$ are general coefficients. These coefficients can take the values $\alpha \in\{-1,1\}, \quad \beta \in\{-1,0,1\}, \quad \gamma \in\{-1,1\}$. Different types of voltage conveyors can be defined by combining the transfer coefficients (Table.1).

Table 1. Definition of three-port voltage conveyors.

\begin{tabular}{cccccccc}
\hline type & $\alpha$ & $\beta$ & $\gamma$ & type & $\alpha$ & $\beta$ & $\gamma$ \\
\hline VCI+ & 1 & 1 & 1 & IVCI+ & -1 & 1 & 1 \\
VCI- & 1 & 1 & -1 & IVCI- & -1 & 1 & -1 \\
VCII+ & 1 & 0 & 1 & IVCII+ & -1 & 0 & 1 \\
VCII- & 1 & 0 & -1 & IVCII- & -1 & 0 & -1 \\
VCIII+ & 1 & -1 & 1 & IVCIII+ & -1 & -1 & 1 \\
VCIII- & 1 & -1 & -1 & IVCIII- & -1 & -1 & -1 \\
\hline
\end{tabular}

Other types of voltage conveyors were derived from the multiple - port current conveyors [5], [6]. For the practical realization of circuits using voltage conveyors a universal voltage conveyor - UVC [7] that can realize all types of three - port and multiple - port voltage conveyors has been designed in our department. 
For the graphic description of voltage conveyor and the design of new circuit the Mason -- Coates graphs with undirected self-loops are used. The description of linear circuits by signal - flow graphs is based on the graphic representation of mathematical formulas of circuits. The linear circuit can be described by a system of $n$ linear algebraic equations

$$
\mathbf{X}=\mathbf{A Y},
$$

where $\mathbf{Y}$ is the column matrix of the known parameters $y_{1}, y_{2}, \ldots, \mathrm{y}_{\mathrm{n}}$, symbol $\mathbf{X}$ stands for the column matrix of unknown parameters $x_{1}, x_{2}, \ldots, \mathrm{x}_{\mathrm{n}}$. Symbol $\mathbf{A}$ denotes the square matrix of coefficients $a_{\mathrm{ij}}(i, j=1,2, \ldots \mathrm{n})$. Equation (2) can be displayed in the graph in many ways that lead to various types of graphs [1]. The Mason - Coates graphs with undirected self-loops are the graphical representation of equation (2) formulated in the form

$$
a_{i i} x_{i}=y_{i}-\sum_{\substack{j=1 \\ j \neq i}}^{n} a_{i j} x_{j} . \quad(i=1,2, \ldots, \mathrm{n})
$$

The system of equations (2) can be displayed in the graph as follows. We display all parameters in the graph by ringlets that are called nodes. The relations between parameters are displayed by oriented lines that are called branches. The transfer of each branch is given by coefficient $a_{\mathrm{ij}}$. Coefficients $a_{\mathrm{ii}}$ on the left side of equation (3) represents transfer of undirected self-loops which belong to the nodes $x_{\mathrm{i}}$. In signal flow graphs representing linear circuits we can start from any system of independent equations (impedances, admittances). For circuits with voltage conveyors it is suitable to start from the system of admittance equations. The branches in a graph will correspond to node voltages in the circuit. The transfers of oriented branches will be given by the sum of admittances between a given pair of nodes while the transfers of undirected self-loops will be equal to the sum of admittances connected to given node. However, the voltage conveyors are irregular active elements. Because of this their signal - flow graphs must be created with the help of hybrid equations [8]. The GVC element can be described by the system of equations

$$
\begin{gathered}
Y_{x} V_{x}=I_{x}, \\
Y_{y} V_{y}=I_{y}, \\
Y_{z} V_{z}=I_{z}, \\
1 . I_{x}=\alpha I_{y}, \\
0 . I_{y}=\beta V_{x}-V_{y}, \\
0 . I_{z}=\gamma V_{x}-V_{z},
\end{gathered}
$$


Equations (4) to (6) describe the properties of the voltage conveyor from the viewpoint of external circuit. Because of this they are written in the form of (3) and correspond to node voltages on GVC terminals. The transfers of the self - loops of these nodes are given by the sum of admittances connected to the given node. Equations (7) to (9) describe the voltage conveyor properties and belong to the internal nodes of the GVC signal - flow graph. The final GVC signal - flow graph is shown in Fig. 2.

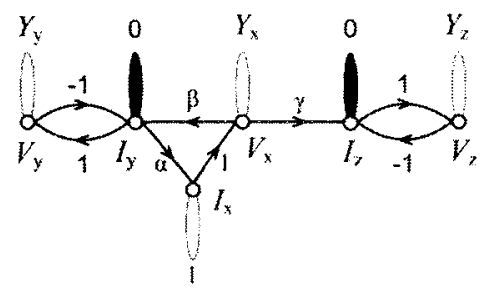

Fig. 2. Full signal -- flow graph of the GVC

The graph in Fig.2 describes all properties of the GVC element. However, it is not suitable to design new circuits. This graph can be simplified by removing node $I_{x}$. This node will be used only in the case that the current $I_{x}$ is the output value of the designed circuit. By removing this node from the graph only the transfer between node $V_{x}$ and internal node $I_{y}$ is changed. Another modification of the GVC graph is based on the idea that the voltage output has an ideally infinite impedance. Because of this, the output port voltage does not depend on the loading impedance. According to this idea the output terminal can be represented in the graph only by one voltage node. The self-loop transfer of this node is at all times equal to one. From the viewpoint of external circuit the branches can only get out of the node $V_{z}$. This property can be with advantage used in the design of new circuits. The simplified GVC signal - flow graph is shown in Fig.3.

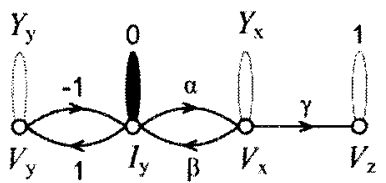

Fig. 3. Simplified signal - flow grapf of the GVC

Just as the voltage output properties can be used in the graph, the properties of the current input which has ideally zero impedance can be used too. In the graph it is possible to display the current input by one current node with self-loop transfer always equal to one. Conversely the branches from an external circuit can only enter into the current node. In the case of voltage conveyors this modification can be used only in second $\cdots$ generation voltage conveyors $(\beta=0)$. A simplified graph of the second - generation voltage conveyors is shown in Fig. 4.

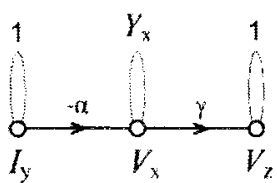

Fig. 4. Simplified graph of second - generation voltage conveyors 


\section{Frequency filter design using signal flow graphs}

The signal - flow graphs can be used to design general circuits with the required transfer function in the frequency filter design. For this purpose Mason's rule for evaluating circuit quantities from a signal - flow graph is used [2].

$$
\frac{x_{i}}{y_{j}}=\frac{1}{\Delta} \sum_{k=1}^{n} P_{k} \Delta_{k},
$$

where

- $x_{i}$ is output quantity

- $y_{j}$ is input quantity

- $P_{k}$ is the transfer of the $\mathrm{k}^{\text {th }}$ forward path from input node $\left(x_{i n}\right)$ to output node $\left(x_{\text {out }}\right)$

- $\Delta$ is the product of all self-loops

- (sum of loop gain products of all possible sets of nontouching oriented loops taken two at a time)

+ (sum of loop gain products of all possible sets of nontouching oriented loops taken three at a time)

- $\Delta_{\mathrm{k}}$ is the value of $\Delta$ for that portion of the graph which does not touch the $\mathrm{k}^{\text {th }}$ forward path.

The graphs of generalized elements that have the possibility of choosing the transfer coefficients can be used with advantage to design circuits with voltage conveyors. However, the biggest advantage in the design of new circuits consists in the properties of the nodes $V_{x}\left(I_{y}\right)$. From the signal - flow graph theory it is known that by adding a general admittance between a pair of nodes $(a, b)$ two oriented branches originate. One from node $a$ to node $b$ and another in the opposite direction. The self-loop transfers of these nodes are changed too. If the general admittance is added between any node in the graph and the node $\mathrm{V}_{\mathrm{z}}\left(\mathrm{I}_{\mathrm{y}}\right)$ only one branch originates and the selfloop transfer of the node $V_{z}\left(I_{y}\right)$ is not changed.

The application of signal - flow graphs will be shown in the multifunctional frequency filter design. Multifunctional circuits are multiple - port networks realizing more types of transfer functions. A multifunctional circuit with one output and more inputs will be designed. Various types of transfer function will be realized by changing the input port without changing the topology and passive elements of the circuit.

From Mason's Rule it is evident that by changing the input node only the transfer function numerator is changed. The expression for the transfer function denominator is given by the determinant of the whole graph and it is the same for all transfer functions. So the multifunctional filter design can be divided into two parts. In the first part the basic graph structure will be designed which the required change of the transfer function numerator is reached. In the second part the oriented loops will be created that determine the graph determinant. 
The basic multifunctional filter graph structure (Fig.5) has been designed using two simplified voltage conveyor graphs (Fig.4).

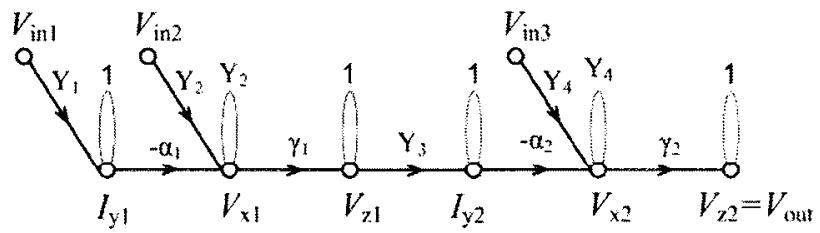

a)

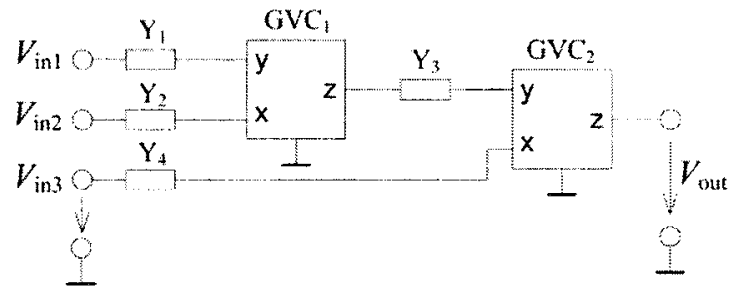

b)

Fig. 5. The basic structure of multifunctional circuit: a) signal - flow graph b) circuit realization

From the graph in Fig. 5 it is evident that by changing the input node the transfers of forward paths between these nodes and the output node are changed. The transfers of forward paths correspond to transfer function numerators and can be expressed by the equations

$$
\begin{gathered}
P_{i n 1}=\alpha_{1} \alpha_{2} \gamma_{1} \gamma_{2} Y_{1} Y_{3}, \\
P_{i n 2} \Delta_{i n 2}=-\alpha_{2} \gamma_{1} \gamma_{2} Y_{2} Y_{3}, \\
P_{i n 3} \Delta_{i n 3}=\gamma_{2} Y_{2} Y_{4} .
\end{gathered}
$$

The next step in the design of the general multifunctional circuit structure is creating oriented loops in the graph. There must be a sufficient number of oriented loops in the graph in order that in the final realization with concrete passive elements the stability criterion will be fulfilled. Oriented loops can be created by adding new branches between any pairs of graph nodes. However, this can give a risk to undesirable forward paths between the input nodes and the output node in the graph which will lead to a change transfer in functions numerators. The specific properties of the nodes $I_{y}$ and $V_{z}$ can be used to create oriented loops. Several possibilities of realizing oriented loops in the graph are shown in Fig.5. The determinants of the graphs are always depicted. Oriented loops can be designed by using voltage conveyors graphs too (Fig.5c, d) 


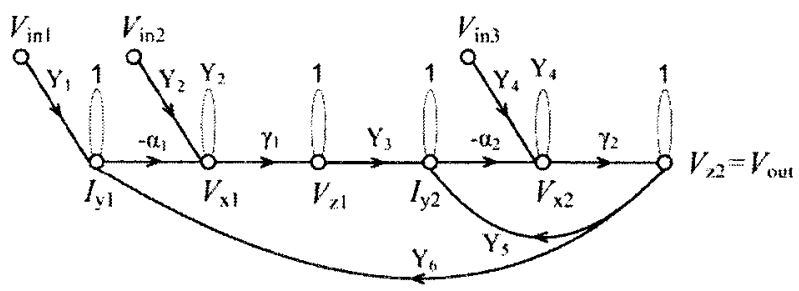

$$
\Delta=Y_{2} Y_{4}+\alpha_{2} \gamma_{2} Y_{2} Y_{5}-\alpha_{1} \alpha_{2} \gamma_{1} \gamma_{2} Y_{3} Y_{6}
$$

a)

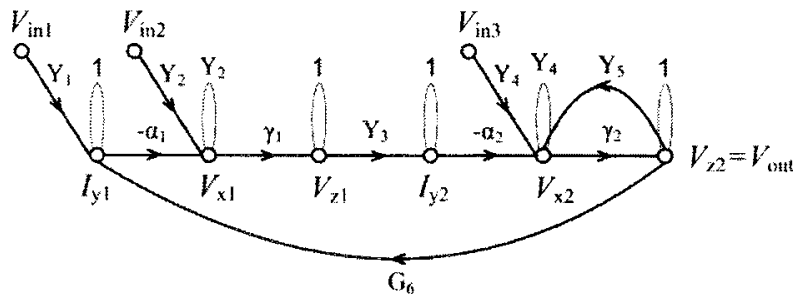

$$
\Delta=Y_{2} Y_{1}+Y_{2} Y_{5}\left(1-\gamma_{2}\right)-\alpha_{1} \alpha_{2} \gamma_{1} \gamma_{2} Y_{3} Y_{6}
$$

b)

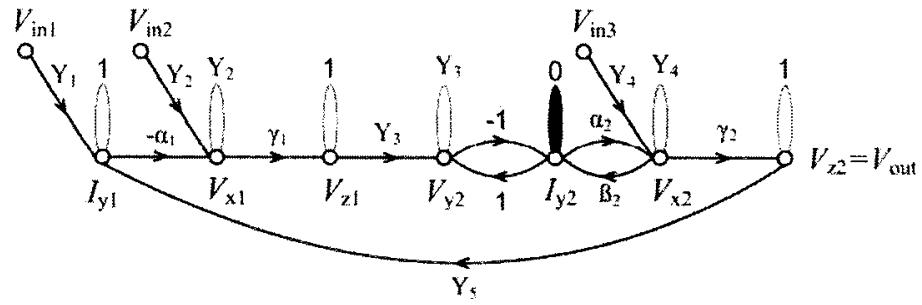

$$
\Delta=Y_{2} Y_{4}-\alpha_{2} \beta_{2} Y_{2} Y_{3}-\alpha_{1} \alpha_{2} \gamma_{1} \gamma_{2} Y_{3} Y_{5}
$$

c)

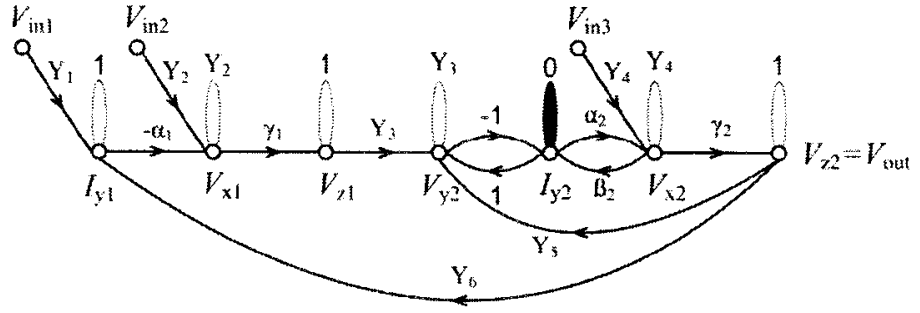

$$
\Delta=Y_{2} Y_{1}-\alpha_{2} \beta_{2} Y_{2} Y_{3}-\alpha_{1} \alpha_{2} \gamma_{1} \gamma_{2} Y_{3} Y_{6}+Y_{2} Y_{5}\left(\alpha_{2} \gamma_{2}-\alpha_{2} \beta_{2}\right)
$$

d)

Fig. 6. General graphs of multifunctional circuits 
The graph in Fig.5c has been chosen for the next design. By choosing particular transfer coefficients the graph determinant has been modified such that all the members of the determinant have the positive sign. In this way the final circuit stability has been achieved. Possible combinations of the concrete transfer coefficients are given in Table 2.

Table 2. Choice of the transfer coefficients

\begin{tabular}{ccccccccc}
\hline variant & $\alpha_{1}$ & $\beta_{1}$ & $\gamma_{1}$ & $\alpha_{2}$ & $\beta_{2}$ & $\gamma_{2}$ & GVCl $_{1}$ & GVC $_{2}$ \\
\hline 1 & -1 & 0 & 1 & 1 & -1 & 1 & IVCII+ & VCIII+ \\
2 & -1 & 0 & -1 & 1 & -1 & -1 & IVCII- & VCII- \\
3 & 1 & 0 & -1 & 1 & -1 & 1 & VCII- & VCIII+ \\
4 & 1 & 0 & 1 & 1 & -1 & -1 & VCII+ & VCII- \\
5 & 1 & 0 & 1 & -1 & 1 & 1 & VCII+ & IVCI+ \\
6 & 1 & 0 & -1 & -1 & 1 & -1 & VCII- & IVCI- \\
7 & -1 & 0 & 1 & -1 & 1 & -1 & IVCII+ & IVCI- \\
8 & -1 & 0 & -1 & -1 & 1 & 1 & IVCII- & IVCI+ \\
\hline
\end{tabular}

The last step in the multifunctional frequency filter design is choosing concrete passive elements. Several possibilities are offered again here. For example $Y_{1}=1 / R_{1}$, $Y_{2}=1 / R_{2}, Y_{3}=p C_{1}, Y_{4}=p C_{2}, Y_{5}=1 / R_{3}$ can be chosen. The final realization of the multifunctional frequency filter is shown in Fig.6.

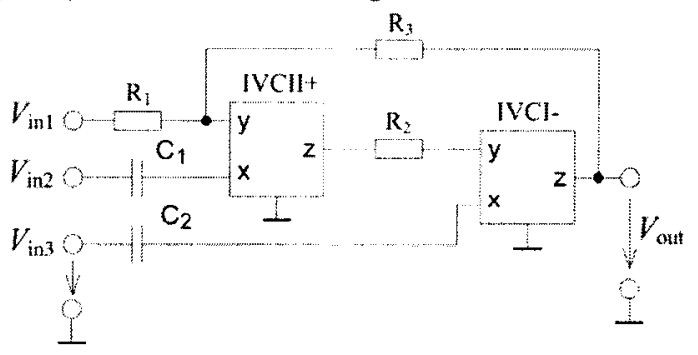

Fig. 7. Designed multifunctional frequency filter

The circuit in Fig. 6 realizes frequency filters of the type of low pass, band pass and high pass with transfer functions (15) to (17):

$$
\begin{gathered}
K_{V, L P}=\frac{V_{o u t}}{V_{\text {in } 1}}=-\frac{R_{3} / R_{1}}{1+\boldsymbol{p} C_{1} R_{3}+\boldsymbol{p}^{2} C_{1} C_{2} R_{2} R_{3}} \\
K_{V, B P}=\frac{V_{o u t}}{V_{\text {in } 2}}=-\frac{\boldsymbol{p} C_{1} R_{3}}{1+\boldsymbol{p} C_{1} R_{3}+\boldsymbol{p}^{2} C_{1} C_{2} R_{2} R_{3}} \\
K_{V, H P}=\frac{V_{o u t}}{V_{i n 3}}=-\frac{\boldsymbol{p}^{2} C_{1} C_{2} R_{2} R_{3}}{1+\boldsymbol{p} C_{1} R_{3}+\boldsymbol{p}^{2} C_{1} C_{2} R_{2} R_{3}}
\end{gathered}
$$


By the connection of inputs $V_{\text {in } 1}$ a $V_{\text {in } 3}$ the band rejection can be realized too (18).

$$
K_{V, B R}=\frac{V_{o u t}}{V_{i n 1}+V_{i n 3}}=-\frac{R_{3} / R_{1}+\boldsymbol{p}^{2} C_{1} C_{2} R_{2} R_{3}}{1+\boldsymbol{p} C_{1} R_{3}+\boldsymbol{p}^{2} C_{1} C_{2} R_{2} R_{3}} .
$$

\section{Simulations}

Using the OrCad -- Pspice simulation program the multifunctional frequency filter was analyzed. The universal voltage conveyor was used as an active element. The frequency filter was designed for characteristic frequency $\omega_{0}=1 \mathrm{MHz}$ and quality factor $\mathrm{Q}=0,707$ (Butterworth approximation). Analyzed amplitude frequency responses of the designed multifunctional filter are shown in Fig. 8.

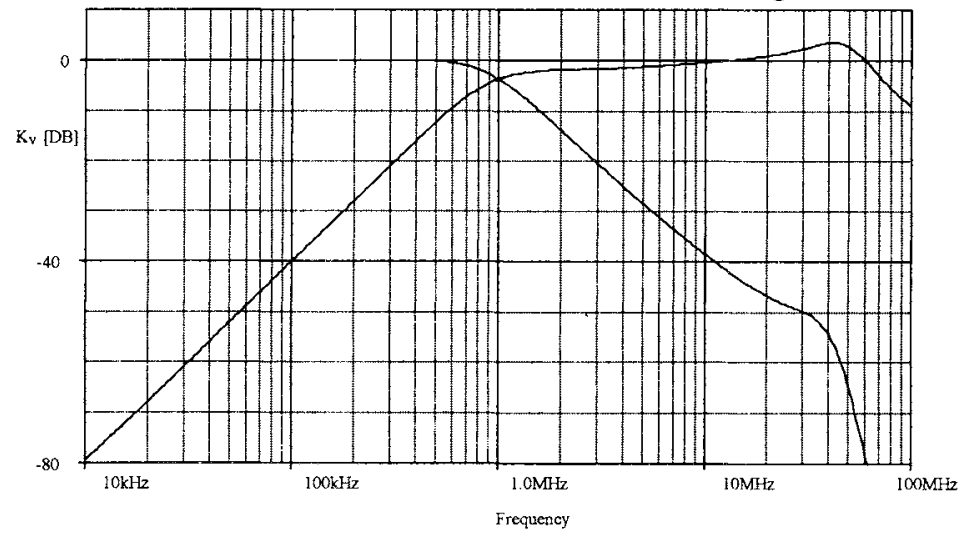

a)

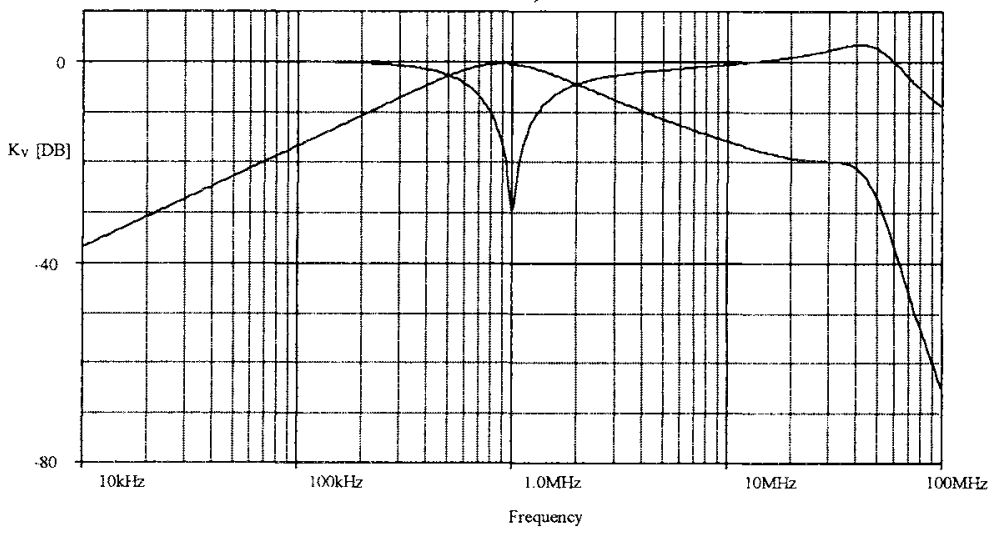

b)

Fig. 8. Analyzed magnitudes of transfer functions a) low - pass, high - pass

b) band - pass, band - rejection 


\section{Conclusion}

The method of active frequency filter design with voltage conveyors using signal flow graphs was introduced in this paper. The multifunctional circuit that can realize low pass, band pass, high pass and band rejection filters was designed using this method. The same procedure of the circuit design can be used for the other types of active elements.

Acknowledgments. The research into new active elements is supported by The Grant Agency of the Czech Republic, project No. 102/06/1383.

\section{References}

1. Wai-Kai Chen.: Circuits and Filters Handbook 2nd Edition. CRC Press, London (2003)

2. Biolek, D., Biolkova,V. Novel Signal Flow Graphs of Current Conveyors. In Proceedings of the International Conference on 38th MWSCAS. Rio de Janeiro (1995) 1058-1061

3. Acar, C., Ozoguz, S.: A new versatile building block: current differencing buffered amplifier suitable for analog signal - processing filters. Microelectronics Journal, Vol. 30 (1999) 157 160

4. Becvar, D.: Voltage Conveyors. Elektrorevue.cz, No. 51, 2001, (in Czech).

5. Vrba, K., Cajka, J. Universal network using DVCC elements for filter realization. In Proceedings of Conf. Telekomunikace 98, Brno (1998) 136-137

6. Vrba, K., Cajka, J.: Universal filter design using the general four-port current conveyor. In Proceedings of Conf. TSP 2000, Brno (2000) 16-19

7. Minarcik, M., Vrba, K.: Low-output and high-input impedance frequency filters using universal voltage conveyor for High-Speed Data Communication Systems. In Proc of Int. Conf. on Networing ICN 2006, Mauritius (2006)

8. Biolek, D., Cajka, J.,-Biolkova, V.: Modeling of current and voltage conveyors by flow graph technique. IASTED, Mexico (2003) 140-145 